\title{
Représentations de la ville informelle dans la littérature hispano-américaine: Santiago du Chili vu par Lemebel et Sutherland
}

LIONEL SOUQUET

> HCTI, Université de Bretagne Occidentale - Brest. Professeur d'espagnol à I'UBO, spécialiste de littérature hispano-américaine des XXe et $X X{ }^{e}$ siècles. Il travaille notamment sur les Cubains Reinaldo Arenas et Abilio Estévez, les Argentins Copi et Manuel Puig, le Chilien Pedro Lemebel et le Colombien Fernando Vallejo. II s'intéresse aux problématiques de la postmodernité, de la marginalité, du genre, du kitsch, de la ville et propose une lecture deleuzienne des textes. lionelsouquet@hotmail.com

Universidad de Valparaíso

Facultad de Arquitectura

Márgenes

Espacio Arte Sociedad

Représentations de la ville informelle dans la littérature hispa-

no-américaine: Santiago du Chili vu par Lemebel et Sutherland

Julio 2013, Vol. 10, Nº 12

pp. 15-22

ISSNN 0718-4034

Recepción: Agosto 2012

Aceptación: Diciembre 2012

\section{RÉSUMÉ}

L'auteur de cet article propose d'aborder le thème de l'informalité urbaine dans la littérature hispano-américaine. Il existe, en effet, dans la littérature de langue espagnole, une longue tradition réaliste où les auteurs conjuguent la fiction à la réflexion philosophique, sociologique, anthropologique, dans une approche documentaire et analytique du réel. Dans une région qui concentre certaines des plus grandes mégapoles mondiales, le regard sur la ville et ses enjeux socio-culturels et politiques est particulièrement aigu. Les Chiliens Pedro Lemebel et Juan Pablo Sutherland sont très représentatifs de cette approche quasi ethnologique du milieu urbain. Mais, tandis que Lemebel propose une sorte d'archéologie néo-picaresque de la pauvreté, Sutherland actualise la figure du pauvre dans celle du marginal qui mine la ville formelle par une pratique informelle.

MOTS CLES

littérature, P. Lemebel, J. P. Sutherland, Santiago du Chili.

Representation of the informal city in the Hispano-American literature: Santiago, Chile according to Lemebel and Sutherland ABSTRACT

The author of this article proposes to address urban informality in the Hispano-America literature. In fact, in Spanish speaking literature there is a long realistic tradition in which authors combine fiction and philosophical, sociological and anthropologic reflection, in a documental/ry and analytical approach to reality. In a region that brings together some of the biggest world megalopolis, the views over political and socio-cultural issues are particularly sharp. Chileans Pedro Lemebel and Juan Pablo Sutherland clearly represent this almost ethnologic approach to urban space. Lemebel proposes a kind of neo-picaresque archeology of poverty, while Sutherland updates the figure of the poor, replacing it for the figure of the marginal who attacks the formal city through its informal activities. KEY WORDS

literature, P. Lemebel, J.P Sutherland, Santiago, Chile

\section{Representaciones de la ciudad informal en la literatura hispano americana: Santiago de Chile según Lemebel y Sutherland RESUMEN}

El autor de este artículo propone abordar el tema de la informalidad urbana en la literatura hispano americana. En efecto, existe en la literatura de habla hispana, una larga tradición realista donde los autores conjugan la ficción con la reflexión filosófica, sociológica, antropológica, en una aproximación documentalista y analítica de lo real. En una región que concentra algunas de las más grandes megápolis del mundo, la mirada sobre los temas socio-culturales y políticas son particularmente incisivas. Los chilenos Pedro Lemebel y Juan Pablo Sutherland representan fielmente esta aproximación casi etnológica del espacio urbano. Por una parte, Lemebel propone una suerte de arqueología neo-picaresca de la pobreza, Sutherland actualiza la figura del pobre en la del marginal que atenta contra la ciudad formal por sus prácticas informales.

PALABRAS CLAVES

literatura, P. Lemebel, J. P. Sutherland, Santiago de Chile 
La forme d'une ville change plus vite, on le sait, que le cœur d'un mortel.

Gracq, 1985, p. 1

La Habana nunca es igual y siempre es igual.

Estévez, 1997, p. 21

Lorsque nous parcourons une ville nous en faisons l'expérience concrète - spatiale et physique- mais nous faisons aussi marcher notre imagination, nous échafaudons des hypothèses sur les gens que nous croisons et les lieux que nous traversons ou ceux où nous n'allons et n'irons jamais. Le philosophe et anthropologue argentin Néstor García Canclini pense les villes contemporaines comme des mégalopoles constituées non seulement d'espaces matériels mais aussi d'espaces symboliques et imaginaires en relation avec les origines multiculturelles de leurs habitants: Une grande partie de ce qui nous arrive est imaginaire, parce que cela ne surgit pas d'une interaction réelle. [...] Les imaginaires ont nourri toute l'histoire de l'espace urbain. (Canclini, 2005, p. 89)1 . Mais, dans un geste typiquement postmoderne, Canclini va encore plus loin dans la "déterritorialisation" de sa discipline puisqu'il s'appuie sur un article de la spécialiste de littérature hispano-américaine Rosalba Campra, La ciudad en el discurso literario ${ }^{2}$, pour affirmer que les villes se fondent aussi dans les livres ou à partir de livres. En d'autres termes: l'identité d'une ville se construit autant sur la représentation littéraire qu'on en donne que sur sa réalité physique, matérielle, ethnologique et sociale. D'un point de vue non plus sociologique mais purement littéraire, l'écrivain Olivier Rolin exprime très élégamment ce lien entre ville et littérature, dans un joli recueil de textes intitulé Sept villes:

Je ne sais pas pourquoi Vieira da Silva faisait des villes avec des caractères de machine à écrire, des amis pourraient me renseigner, mais à quoi bon? De toute façon, je trouve qu'elle avait raison. Et pas seulement parce que Alexandrie c'est Durrell, comme on dit, ou Cavafy pour les Grecs, ou Trieste Svevo, ou Prague Kafka, etc. Que veut dire, d'ailleurs, cette équivalence? Probablement que les écrivains, avec leurs perspectives et leurs avenues et leurs quais de mots, leurs coupoles et leurs colonnes de mots, et aussi leurs poubelles, égouts, remugles, papiers gras de mots, nous aident puissamment à nous tromper, à errer dans et sur les villes. On lit un de ces livres dont une ville est le lieu et puis, débarquant un jour pour la première fois, on constate que rien n'a changé depuis qu'on n'y est jamais allé. (Rolin, 1988, pp. 7-8).

L'ambition de notre travail, sans prétentions sociologiques ni urbanistiques, est de montrer que la littérature porte souvent un regard extrêmement pertinent sur les villes. Les cultures hispaniques et hispano-américaines n'ayant pas développé de grande tradition philosophique -contrairement à l'Allemagne, à la France, à la Grande-Bretagne ou aux Etats-Unis- cette réflexion a généralement été prise en charge et portée par la littérature. II existe donc, depuis le XVle siècle, dans la fiction en langue espagnole, une profonde tradition réaliste comme en témoignent le roman picaresque ou les prétentions documentaires et scientifiques de la littérature réaliste et naturaliste du XIXe siècle. La fiction a souvent permis aux écrivains de diffuser leurs idées philosophiques, religieuses, politiques ou sociales en contournant la censure de l'Inquisition ou des régimes politiques autoritaires. Or, les thèmes de la ville et de la pauvreté -omniprésents en Amérique latine et conjugués au sein de la ville informelle- ne pouvaient laisser les écrivains indifférents. En effet, comme le rappelle Ana Claudia Correa Díaz, en Argentine, en Uru- guay et au Chili l'urbanisation a commencé très tôt, dès les années 1920 et 1930. Dans certains pays comme l'Argentine, le Brésil, le Chili, le Mexique, le Pérou, I'Uruguay et le Venezuela, I'urbanisation est supérieure à $70 \%$ et, selon les études des Nations Unies réalisées en 2001, la population de la ville informelle représenterait 35,5\% des urbains (Correa Diaz, 2007, pp. 4-18). Correa Díaz note aussi que [...] ni le secteur formel ni le secteur informel n'ont des caractéristiques exclusives, ce qui rend difficile leur identification et leur nette différenciation. [...] L'informalité [emploi, logement, transport] est un symptôme de la faiblesse des États et du manque d'offre dans le secteur formel [...] (Correa Diaz, 2007, p. 10)

Après avoir évoqué brièvement quelques exemples pris chez l'Argentin Manuel Puig, le Cubain Reinaldo Arenas ou le Colombien Fernando Vallejo, nous nous arrêterons un peu plus longuement sur deux écrivains chiliens qui évoquent, dans leurs écrits, deux approches différentes du Santiago informel.

Le Chilien Pedro Lemebel, né en 1955 dans une famille très modeste, a fait son apparition dans le paysage artistique de la fin de la dictature de Pinochet en tant que co-fondateur des Yeguas del Apocalipsis, un petit groupe de plasticiens qui réalisaient des performances extrêmement provocatrices. Lemebel s'essaie ensuite à l'écriture et devient, par ses publications régulières dans des revues satiriques, le chroniqueur le plus en vue du pays, détesté par la droite mais adulé par une grande partie de la gauche. Malgré son amitié avec Gladys Marín, présidente et secrétaire générale du Parti Communiste Chilien, Lemebel gardera toujours une distance critique vis à vis du PC, publiant notamment un Manifeste (intitulé Manifiesto) dans lequel il s'affirme comme militant homosexuel queer et activiste du sida -héritier de Pasolini et de Ginsberg- et où il met la gauche en garde contre un manque de tolérance à l'égard des différences sexuelles et identitaires. Cette relation problématique entre homosexualité, engagement politique et révolution est reprise, selon une approche fictionnelle, dans son seul roman publié à ce jour, Tengo miedo torero (sorti en 2001 et traduit en français sous le titre Je tremble, ô matador, en 2007), un hommage au Baiser de la femme araignée (El beso de la mujer araña, 1976) de l'Argentin Manuel Puig. Lemebel est un artiste protéiforme et talentueux qui se met constamment en scène dans son œuvre et dans la vie médiatique, non par auto-complaisance narcissique mais en tant que fils de pauvres, métisse et homosexuel, comme emblème des dominés sociaux. Malgré le succès de son roman, Lemebel reste avant tout un chroniqueur profondément ancré dans la réalité sociale et politique de son pays. Nous verrons donc comment cet artiste articule l'évocation du bidonville dans lequel il a passé sa petite enfance avec une réflexion intime et originale sur le déterminisme social.

C'est dans une perspective apparemment plus "sociologique" ou "anthropologique" que littéraire que je propose d'aborder ensuite les nouvelles du "jeune" écrivain chilien Juan Pablo Sutherland (né en 1967), Ángeles negros $(1994)^{3}$-dont la publication suscita un intense débat sur la création artistique et la censure dans le Chili de la post-dictature ${ }^{4}$ - à la lumière des travaux théoriques de Canclini sur les imaginaires urbains. Les concepts développés par l'anthropologue se retrouvent sous une forme littéraire, non théorique, comme en creux, dans les nouvelles de Sutherland qui, de son côté, donne le plus souvent à son œuvre littéraire valeur de document et de manifeste. Etonnamment, la lecture théorique de Canclini sur les villes nous aide aussi à évaluer le degré de réalisme des nouvelles de Sutherland et à en décrypter toute la portée idéologique, au-delà de l'apparente futilité des personnages et des 
situations. II faut dire que Sutherland -admirateur de Lemebelest un remarquable observateur des pratiques (sub-)culturelles et informelles des "citadins postmodernes" et ce n'est pas étonnant quand on sait qu'il est lui-même essayiste, théoricien de la littérature et chroniqueur engagé.

\section{CENTRE ET PERIPHERIE, GUEUX ET PICAROS}

L'enquête des Nations Unies réalisée en 1977 sur la situation du logement dans le monde définit les "habitations improvisées" en tant que toute installation de fortune ou construction faite de différents matériaux de récupération et bâtie sans plan déterminé pour servir d'habitation, utilisée comme habitation par un ménage au moment du recensement. Ce type d'unité se rencontre habituellement dans les zones urbaines et suburbaines... (Hardoy et Satterthwaite, 1981, p. 288) ${ }^{5}$. La ville informelle est donc traditionnellement -et logiquement- associée à la notion de pauvreté. Or, la figure du pauvre occupe une place privilégiée dans la tradition littéraire espagnole et hispano-américaine où elle apparaît sous les traits du pícaro, le gueux. Les romans picaresques les plus célèbres sont Vida de Lararillo de Tormes y de sus fortunas y adversidades ${ }^{6}$ (œuvre anonyme publiée en 1554), Guzmán de Alfarache ${ }^{7}$ (1599) de Mateo Alemán et La vida del Buscón ${ }^{8}$ (1626) de Quevedo. Le genre (et ses différents avatars) -fondateur d'une puissante tradition réaliste- survivra dans la péninsule et dans les colonies hispano-américaines jusqu'au XIXe siècle et trouvera un nouvel essor, à partir du milieu du XXe siècle, sous le terme de néo-picaresque. Le personnage central, le pícaro, est (presque) toujours de basse extraction, sorti des bas-fonds ou du ruisseau (parfois au sens propre, comme nous le verrons): ses parents, ouvertement marginaux ou délinquants, représentent la lie de la société mais expriment le désir de arrimarse a los buenos, c'est-à-dire de s'élever. Parents et enfants sont de la caste des "sans honneur", dans une Espagne du Siècle d'Or dont les valeurs suprêmes sont la naissance et la "pureté de sang" ${ }^{9}$ (prérequis d'honorabilité) mais où l'argent est, en fait, le moteur réel. Le pseudo ascension sociale du pícaro, qui se fait par des moyens illégitimes tels que le vol ou l'escroquerie et le mène parfois de l'abri de fortune aux plus somptueux palais ou en prison, est une négation comique des valeurs de la société espagnole des XVle et XVIle siècles. Souhaitant dénoncer les injustices sociales, particulièrement flagrantes dans l'inégalité des droits au logement, certains écrivains hispano-américains de la deuxième moitié du XXe siècle ont donc choisi assez "naturellement" le genre néo-picaresque afin de dépeindre, selon une perspective typiquement postmoderne, la réalité quotidienne des pauvres.

Ainsi, dans son très célèbre roman Boquitas pintadas (1969), l'Argentin Manuel Puig (Argentine, 1932 - Mexique, 1990) décrit avec une précision radioscopique les conditions de vie déplorables des populations pauvres, dans une Argentine des années 19301940 qui préfère afficher les richesses de la grande bourgeoisie des vieux propriétaires terriens et surtout la toute nouvelle réussite de la classe moyenne issue de la grande vague d'immigration (1880-1930), souvent indifférente à la pauvreté des masses, les cabecitas negras (petites têtes noires), immigrants pauvres et descendants d'anciens gauchos prolétarisés. En marge de l'imposant centre de Buenos Aires, la mégapole cosmopolite, s'étalent les villas miserias, les bidonvilles. Les pimpantes bourgades de la pampa sont, elles aussi, bordées de rues en terre où les nombreux laissés pour compte du miracle argentin tentent de se bricoler un abri, loin des yeux du medio pelo, la classe moyenne.
La pompe était à l'autre bout du terrain où se trouvait la cabane, il se mouilla le visage et le cou, se rinça la bouche. [...] il entra dans la chambre pour enfiler son bleu de travail. Dans un grand lit il y avait ses deux sœurs. [...] Le sol était de terre battue, les murs de pisée, le toit de tôle. [...] La cuisine n'était pas tout à fait terminée. Pancho l'avait commencée avec des matériaux de démolition. (Puig, 1972, p. 77).

Le narrateur à la première personne de Celestino antes del alba (El pozo, 1967) et El palacio de las blanquísimas mofetas (publié clandestinement en 1980 mais écrit dans les années 60), les deux premiers romans du Cubain Reinaldo Arenas (Holguín, Cuba, 1943 - New York, USA, 1990) -romans de l'enfance et de l'adolescence fortement autobiographiques dont l'action se situe dans les années 1940-1950, sous la dictature de Batista- passe du dénuement de la cabane de paysans pauvres où il est né à une maison de ville presque plus misérable encore, mélangeant curieusement les apparences de l'urbanité et les prémices d'un modeste embourgeoisement avec les contingences de la pauvreté, de la précarité et l'absence de commodités traditionnellement associées à l'habitat urbain formel. Cette masure-égout n'a rien à envier à la maison " lugubre et obscure " de l'écuyer, dans le troisième traité du Lazarillo:

Un couloir étroit avec une fenêtre donnant sur le mur de l'usine. [...] Une salle à manger sans cloisons où les mouches et le soleil déferlent sans cesse. La maison se termine par une petite cour, réduit où s'accumulent tous les déchets ainsi que les eaux usées de la cuvette et du lavabo. (Arenas, 2001, p. 156) ${ }^{10}$.

L'aspect picaresque des origines sociales est particulièrement net chez le Chilien Pedro Lemebel, non seulement chez la Loca del Frente, le protagoniste principal de son roman Tengo miedo torero (2001) et son double littéraire, mais aussi dans les chroniques où il se met lui-même en scène. Les souvenirs d'enfance de Lemebel sont associés au Zanjón de la Aguada, un grand canal qui, depuis des générations, sert d'égout à la ville de Santiago et le long duquel s'est peu à peu développé una callampa, un bidonville tristement célèbre, détruit pas un incendie en 1958 et reconstruit sous le nom de población La Victoria, sur un terrain public, en préfabriqués agrémentés de quelques infrastructures sanitaires, grâce à l'aide d'une communauté religieuse. Cette "épopée" de la misère et de la précarité avait été filmée par Rafael Sánchez: Las callampas (1958), produit par l'Institut filmique de I'Université Catholique du Chili et considéré comme le premier documentaire social du cinéma chilien. Mais Lemebel laisse entendre implicitement que ce film, en mettant l'accent sur la reconstruction et sur l'élan de solidarité généré par l'œuvre de Bienfaisance publique Hogar de Cristo, fondée en 1944 par les Jésuites, mythifie partiellement la réalité et en gomme les aspects les plus sordides:

Mais le Zanjón, plus qu'un simple mythe de la sociologie urbaine, fut une ruelle longeant le fatidique canal du même nom. Une rive marécageuse où à la fin des années quarante se sont installées, peu à peu, des planches, du papier bitumé, des cartons et, du jour au lendemain, les maisons étaient prêtes. [...] Les masures précaires fleurissaient parmi les ordures. [...] le problème du logement a toujours été une excursion aventureuse pour les dépossédés [...] au Zanjón, il fallait aller chercher loin l'eau pour boire, cuisiner ou se laver, là où un bassin ouvert en permanence servait à alimenter le bidonville. De la même façon, l'évacuation des eaux usées se résumait en une rigole puante qui coulait parallèlement au campement, où les femmes jetaient les bouillons fétides des excréments. (Lemebel, 2004, pp. 13-16) ${ }^{11}$. 
Cette origine sociale extrêmement défavorisée, ainsi que les caractéristiques topo-(et hydro-)graphiques du quartier de son enfance (le Zanjón fait écho à la rivière Tormes), dont Lemebel dresse un tableau misérable mais haut en couleurs, le rapprochent étonnamment du personnage de Lazarillo:

Ma naissance se fit dans la rivière Tormès, raison pour laquelle je pris ce nom; [...] et alors qu'une nuit ma mère était au moulin, enceinte de moi, elle perdit les eaux et me mit au monde à cet endroit. C'est pourquoi, en vérité, je peux dire que je suis né dans la rivière. (Rico, 1990, pp. 12-14) ${ }^{12}$.

Chez Lemebel comme chez Arenas, l'infiltration des eaux fétides conjugue la «maison" ou, plus exactement, la masure au schème du cloaque. Le Zanjón putride de Lemebel rejoint les délires scatologiques de la picaresque du grand Quevedo. Dans une autre chronique, où le Chilien évoque sa vie d'adulte dans les banlieues pauvres de Santiago, le fleuve dégradé en égout trouve son envers dans une entrée d'immeuble squattée par de jeunes loubards, sous les apparences d'une flaque d'urine dégoulinante transcendée, par les envolées lyriques de Lemebel, en une cascade d'amour aux "parfums" de Zanjón: [...] m'injectant alors le bord argenté de l'urine qui descend nue les marches jusqu'à l'endroit où elle forme une étoile fumante. Moi je fume ces vapeurs dans un soupir d'amour pour son exil rebelle. (Lemebel, 2004, p. 29) ${ }^{13}$. En fait, comme l'exprime magnifiquement Arenas, que son passé soit glorieux ou "crotteux", le néo-picaro est un nostalgique presque proustien de la jeunesse et de l'enfance: Maintenant, même les lieux les plus détestables, sachant qu'ils étaient désormais perdus, se transformaient en lieux vénérables. (Arenas, 2001, p. 184) ${ }^{14}$.

Par un autre renversement comique, Lemebel, chroniqueur de sa propre enfance, se présente comme un niño guarisapo, être amphibien, enfant-batracien qui "accouchera" lui-même, dans une diarrhée fleurie comme l'eau d'un marais (florida diarrea como agua de pantano), d'un têtard ingurgité avec la boue du Zanjón. Dans l'émouvante chronique de ses origines, Lemebel ajoute:

On dirait qu'à l'évocation de cet hier, la tremblante matinée infantile aurait tatoué avec de la glace sèche la peau de mes souvenirs. [...] Dans ce fouillis d'odeurs pourries et de fumées de sciures, "j'ai appris tout ce qu'il y avait de bon et j'ai su le mal de toute chose ${ }^{15}$, j'ai connu la noblesse de la main humble et j'ai peint ma première chronique avec les couleurs de la boue où tourbillonnait le lait trouble de ce Zanjón. (Lemebel, 2004, p. 15) ${ }^{16}$.

La pauvreté et l'adversité apparaissent à la fois comme une école de la vie et une école de l'art, nourrie par la tradition populaire (ici le tango). Nous voyons aussi que, par rapport au genre picaresque, la perspective diffère sur un point essentiel: contrairement à l'auteur des romans de gueuserie (qui était, en fait, un clerc bien éloigné de la réalité quotidienne des pauvres), Lemebel revendique un principe d'identité parfaite entre la figure du miséreux et lui-même. Plus qu'un porte parole du lumpen-prolétariat, Lemebel en est l'émanation, il est la voix du "gueux" chilien. Evoquant avec amour le bidonville où il est né, Lemebel revendique pleinement et fièrement ses origines en leur déniant tout caractère honteux et en soulignant la noblesse des humbles: j'ai connu la noblesse de la main humble. II clame contre le déterminisme réactionnaire, la fierté d'être "né dans le ruisseau" et le mérite d'en être sorti malgré les innombrables barrières socio-culturelles. II dénonce parallèlement l'attitude éhontée des pitucos ${ }^{17}$, les bourgeois snobs et méprisants, qui n'ont jamais hésité - selon lui- à soutenir des dictatures com- me celle de Pinochet pour continuer à exploiter le prolétariat. Alors que le pícaro est -selon le terme de l'hispaniste Maurice Molhoun "infrahumain", le gueux lémébélien est tout simplement un dominé social dont la situation déplorable peut et doit changer par le combat politique. La spécificité et le talent de l'écriture lémébélienne tiennent dans cette faculté à dépeindre une réalité particulièrement crue de façon lyrique mais sans l'atténuer. Les textes de cet artiste proche du Parti Communiste Chilien sont toujours fortement engagés mais sans jamais renoncer à la valeur esthétique, travers fréquent du réalisme social des grands écrivains latino-américains des années 1940, notamment les indigénistes, influencés par le réalisme socialiste alors en vigueur en Union Soviétique. Chez Lemebel c'est, au contraire, le travail de création littéraire associé à un regard ethnologique (empirique mais très aiguisé) qui transcende, dans son œuvre, le discours de dénonciation politique.

\section{CENTRE FORMEL ET PRATIQUES INFORMELLES}

Si l'on doute encore que l'identité d'une ville se fonde autant sur la représentation littéraire qu'on en donne que sur sa réalité physique, matérielle, ethnologique et sociale, la violence des réactions provoquées par la publication de Ángeles negros nous prouve que la littérature a réellement prise sur le réel: elle est un contre-pouvoir redoutable et redouté, surtout lorsqu'elle parle de la ville, de la polis comme espace même du pouvoir. Comme Sutherland l'explique lui-même dans La revuelta de un inicio sexual (Sutherland, 2004, pp. 9-19), le prologue qu'il a écrit pour la réédition de son premier recueil de nouvelles en 2004, la première publication de Ángeles negros, en 1994, avait provoqué un terrible scandale dans le Chili de la transition vers la démocratie: La discussion par rapport à Ángeles negros fut marquée par l'attitude de la droite la plus réactionnaire et le centre politique le plus conservateur, on ne voyait pas d'un bon œil la légitimation, par un financement d'état, d'une thématique aussi aberrante que l'homosexualité. (Sutherland, 2004, pp. 1415). Sutherland avait en effet publié son recueil grâce à un financement du très officiel Fondart (Fondo de las Artes), dépendant du Ministère de l'Education! De plus, cette publication avait réactivé un autre scandale: la bourse, également attribuée par le Fondart à Juan Dávila (peintre chilien dont Sutherland cite le nom et qui illustrera la couverture de son deuxième recueil de nouvelles), auteur d'une carte postale représentant un Simón Bolívar ${ }^{18}$ métisse et transsexuel, qui avait provoqué un incident diplomatique entre le Chili, la Bolivie et le Venezuela, pays dont l'identité est fortement liée à l'image du grand libérateur. Dans ce lourd contexte politique, on comprend mieux les enjeux idéologiques d'un recueil de nouvelles dont le cadre unique et presque obsessionnel est la ville de Santiago. S'il est vrai qu'une œuvre littéraire peut se lire comme un relai, une illustration, du discours ethnologique sur les villes, la littérature va parfois peut-être encore plus loin que le discours scientifique car, dans une certaine mesure, elle modèle la réalité.

Canclini estime qu'il est indispensable de s'intéresser à la multiculturalité de l'espace urbain. En fait, dans une même ville, il en existe souvent plusieurs : le centre historique, bien sûr, mais aussi la ville industrielle qui déterritorialise I'urbain. Un troisième type de ville, propre à la modernité et à la postmodernité, s'est développé: c'est la ville informationnelle et communicationnelle, celle du câble, du fax, des satellites et d'internet. Mais toute cette modernité, cette extraordinaire offre culturelle internationale se heurte souvent, en Amérique latine, aux limites du développement et des infrastructures (vétusté ou désorganisation des voies de communication, pannes d'électricité, etc.) ce qui, selon Canclini, donne à ces villes l'aspect d'un vidéoclip contradictoire et chaotique: la ville latino-américaine vit dans une tension constante entre des 
traditions "de quartier" bien ancrées, qui génèrent un certain type de fonctionnement et de communication urbaine, et une modernité, certes précaire mais réelle, qui inscrit ces villes dans la postmodernité. La ville vidéoclip est une ville qui fait coexister à un rythme accéléré un montage effervescent de cultures de différentes époques. En fait, le concept de ville vidéoclip, utilisé par Canclini pour expliquer la ville postmoderne, est complètement intégré par Sutherland pour écrire cette ville. Ángeles negros relève véritablement de la technique et de l'esthétique du vidéoclip, ce qui en fait d'ailleurs la force et la faiblesse. La structure interne de l'œuvre fonctionne comme un reflet ou une transposition de la réalité des pratiques relationnelles et communicationnelles de la ville postmoderne et, en particulier, des réseaux informels qui se tissent dans le milieu underground.

Selon Ana Claudia Correa Díaz:

[...] dans le champ de l'économie et de l'urbanisme, l'informalité est souvent présentée comme une anti-formalité et non pas comme l'affirmation d'un caractère propre qui indiquerait un mode de faire différent de ceux reconnus par la norme formelle. Cependant, l'opposition entre formalité et informalité n'est pas si nette ni si simple: des situations intermédiaires - ce que nous allons appeler "espaces gris»sont souvent présentes et une lecture dichotomique masque la complexité du phénomène. (Correa Diaz, 2007, p. 5).

Pour Gilles Deleuze et Félix Guattari, la ville, dans sa géométrie et sa segmentarité, est l'espace même du pouvoir. Dans Mille plateaux, ils appuient leur hypothèse sur les observations du grand urbaniste Virilio:

Paul Virilio montre comment l'empire romain impose une raison d'Etat linéaire ou géométrique, qui comporte un dessin général des camps et des places fortes, un art universel de "borner par des tracés", un aménagement des territoires, [...] une transformation du monde en ville, bref une segmentarité de plus en plus dure. (Deleuze et Guattari, 1980 , p. 258) ${ }^{19}$.

II va sans dire que les "centres historiques" -l'essence même de la ville formelle- sont traditionnellement conçus comme une concentration volontairement ostentatoire du pouvoir. Le centre de la ville a donc souvent été -symboliquement ou réellement- interdit aux marginaux. El lugar sin límites (Ce lieu sans limites, 1966), petit chef-d'œuvre du Chilien José Donoso et texte fondateur pour Sutherland, montre bien cette fascination mêlée de peur que la ville exerce sur le travesti la Manuela, figure emblématique de la marginalité:

Bien sûr qu'elle aimerait aller vivre ailleurs et ouvrir un nouveau commerce. Mais elle ne quittait pas la Estación El Olivo parce que c'était un petit village où tout le monde les connaissait, personne ne faisait plus attention à eux, tellement ils étaient habitués. Même pas les gosses, qui le savaient en venant au monde. Pas d'explications à donner, avait dit la Petite Japonaise et un beau matin le village va disparaître et nous deux avec, ce village de merde qui ne demande rien, qui ne s'étonne de rien. (Donoso, 1987, p. 50) 20.

Le très polémique Fernando Vallejo exprime à peu près la même idée, dans El fuego secreto (1985), quand il raconte comment, dans la Colombie des années soixante, son frère et lui entraînaient les garçons loin de la ville pour mieux les séduire:

Mais pourquoi diable emporter les victimes (je veux dire les jeunes gens) aussi haut dans la montagne? L'idée n'était-elle pas trop excentrique? C'est bien ça, en effet, vous l'avez dit: "excentrique", en dehors du centre. Nous les sortions à la périphérie pour couper tous leurs liens avec le monde habituel, pour les débarrasser des tabous, de la ville, de leurs vêtements. (Vallejo, 2004, p. 219) ${ }^{21}$.

Ici, c'est même la ville dans son ensemble qui est assimilée à la notion d'espace formel, normatif. Cependant, dans son célèbre et iconoclaste roman La virgen de los sicarios (La Vierge des tueurs, 1994), l'auteur colombien met en évidence l'existence de deux espaces -formel et informel- dans la même ville: il oppose le centre institutionnel à l'univers marginal et transgressif des sicaires, les tueurs à gages issus des bidonvilles qui s'étalent sur les collines de Medellín. Dans un premier temps, la ville formelle semble envahie par la "barbarie» de l'informel mais Vallejo amène le lecteur à comprendre que la ville institutionnelle n'est que faussement policée: la forme ne serait là que pour masquer le chaos structurel provoqué par l'incurie et le cynisme des classes dominantes! En fait, la ville de Medellín -non seulement métaphore et métonymie de la Colombie mais aussi de l'ensauvagement de la totalité du monde contemporain- n'est pas la proie d'une ville informelle et périphérique qui la grignoterait et la rongerait: elle est principalement victime d'ellemême, c'est-à-dire de la cupidité de ses classes dirigeantes.

L'affrontement "centre-ville-civilisationnel-unique» versus "faubourgsmultiples-fourmillant-de-rufians-et-marginaux" n'a-t-il pas constitué le nœud thétique de la plupart des civilisations urbaines? Mais, comme Canclini, la plupart des ethnologues constatent que, globalement, les usages de l'espace urbain changent et que le centre perd son statut hégémonique: on passe de villes centralisées à des villes multifocales et polycentriques où se développent de nouveaux centres à travers les shopping centers et d'autres types d'urbanisation, populaires ou non, cités dortoirs ou quartiers résidentiels, la population quittant le centre pour différents motifs: Ainsi nous nous resituons dans une ville disséminée, une ville dont nous avons de moins en moins idée d'où elle se termine, où elle commence, où nous nous trouvons. (Canclini, 2005, p. 82)22. Dans les années 1960 et 1970 , notamment, beaucoup de grandes villes occidentales, en Europe ou sur le continent américain, ont vu les classes dominantes déserter les centre-villes pour des quartiers résidentiels de luxe (des country clubs ressemblant parfois à de véritables camps retranchés, en Argentine ou au Mexique) et, par un système de vases communiquants, des populations plus ou moins marginales ont envahi ces centres "dégradés".

Sutherland s'intéresse aux minorités sexuelles, à ce que j'appellerai les "nouveaux libertins postmodernes ${ }^{23}$ " et, en particulier, aux communautés gay et queer ${ }^{24}$, qui contrairement à la Manuela de Donoso ont quitté les marges de la ville pour en investir le centre. Ses personnages, les "Anges noirs", des anges déchus, sont des nomades, des "traversants" dont les parcours sont régis par la consommation d'alcool, de drogue, de sexe et de musique des années 1970 au début des années 90: pop, rock, New wave. Tous ces personnages déambulent dans un Santiago explicitement identifiable $^{25}$. Les personnages deviennent alors les auteurs -parfois inconscients- d'une géographie érotique qui trace des "lignes de fuite" de lieux de rencontres en "lieux de drague", de bars en boîtes. Ces individus vivent et déambulent au sein même de la ville formelle, institutionnelle, la ville du pouvoir et des classes dominantes mais ils en font un usage libre, complètement émancipé des normes de l' "urbanité" bourgeoise, un usage informel. 
La transgression des normes imposées par les "bonnes mœurs" résonne comme un attentat au sens propre. C'est essentiellement par le détournement des espaces publics que les personnages minent et atomisent la segmentarité de la ville, la rendent "moléculaire" au sens deleuzien. Cette ville des sexualités alternatives, ville dans la ville, trouve son centre, ou plutôt une multitude de centres éclatés, atomisés, "moléculaires", dans ces morceaux de "nature domestiquée, apprivoisée", non-ville, espaces de tous les possibles que sont les parcs de Santiago: le célèbre Parque Forestal mais aussi Santa Lucía, Baquedano, Balmaceda ou le cerro de San Cristóbal:

[...] on avait des opportunités sexuelles n'importe où et à n'importe quelle heure, dans le parc Forestal, derrière les Beaux Arts à quatre heures du matin, dans un immeuble du quartier Brasil [...] dans la station de Métro de l'Université du Chili, où on a terminé dans une étreinte passionnelle avec un garde dans l'une des salles donnant sur le quai, ce genre d'endroit qui n'sert jamais à rien. (Sutherland, 2004, pp. 88-89) ${ }^{26}$.

En fait, chaque groupe, presque chaque individu, connaît et expérimente de petites enclaves par lesquelles il transite. Ce sont des parcours microscopiques par rapport à l'ensemble de la ville dont pratiquement personne n'a de perception vraiment globale. Au milieu de la décomposition des mégavilles, Canclini souligne l'importance des micro-espaces (bars, discothèques, etc.), lieux publics investis et privatisés par certains secteurs de la population, espaces semipublics et semiprivés à la fois, qui fonctionnent comme des marqueurs et obligent à reconsidérer la problématique public-privé. De son côté, Sutherland montre aussi une pratique détournée des lieux publics: les bars et discothèques fonctionnent comme clubs gays privés et les parcs et jardins publics qui-dans la journée-- servent d'écrin aux statues des "grands hommes" de la nation et aux monuments officiels, sont investis -la nuit-- par une "faune" marginale qui les "dévie" de leurs "honorables" fonctions, entraînant irrésistiblement la ville formelle vers l'informel. Pendant la Semaine Sainte, les personnages de la nouvelle Como si fuera otro projettent, sur les murs de la cathédrale, des photos de leurs amis gays en train de faire l'amour dans les jardins publics. La sexualité, que la société cantonne dans le domaine du privé et que les régimes autoritaires répriment, est ici affichée, dans ses pratiques les plus transgressives et informelles, au sein même de la ville formelle. Pratiqué en public, l'acte sexuel intime se transcende en geste politique des plus subversifs. L'exhibitionnisme sexuel s'oppose à la monumentalité ostentatoire de la ville. Comme dans La Havane de Reinaldo Arenas, la jouissance effrénée devient une façon concrète de lutter contre le pouvoir et les différentes formes de répression, policière ou simplement morale:

Pendant le premier mois on s'est retrouvés au Prosit, Place Italia. [...] On finissait dans le parc Baquedano à fumer des joins et aux aguets à cause des "forces spéciales antimanifs". Les buissons nous ont toujours aidés. Avec Axel on a eu beaucoup de relations sexuelles en public [...] (Sutherland, 2004, pp. 38-39) ${ }^{27}$.

\section{CONCLUSION}

Comme Stéphanie Decante le montrait dans I'un des tout premiers articles universitaires consacrés à Lemebel, la subculture "folle" redessine et réécrit la géographie d'un Chili de la transition vers la démocratie qui se livre à ce que le sociologue Tomás Moulian appelle une "opération de transformisme", [...] la transmutation d'une politique ouvertement répressive en une autre apparemment consensueIle, marquée par des logiques néolibérales [...] (Decante, 2005, p. $71)^{28}$, un libéralisme marchant de concert avec une morale conservatrice et une terrible amnésie historique et politique que Lemebel dénonce activement. L'une des étapes de cette dénonciation passe par un travail de récupération de la mémoire des sans-mémoire, des humbles, des "gueux". Si l'historiographie, l'histoire des idées et l'abstraction sont l'apanage des classes dominantes, l'écriture archéologique et sentimentale de ce que Deleuze appelle le "peuple mineur" passera donc par la reconstruction de la vie quotidienne et concrète, des pratiques culturelles populaires -voire marginaleset, au premier chef, par l'évocation de l'espace dévolu aux dominés. Or, face à la segmentarité de la ville formelle et normative des puissants, la ville des pauvres -faubourg, "fausse ville", ville à côté de la ville, ville improvisée- semble se caractériser par l'informel. Dans le texte néo-picaresque qu'il consacre au Zanjón de la Aguada, le bidonville de sa petite enfance, Lemebel nous offre une "vision d'en bas" et met en évidence - de façon non théorique mais très précise et évocatrice, comme en creux par rapport aux études anthropologiques et urbanistiques- l'essence même de cette informalité: agglomérats de matériaux de récupération et liquidité, viscosité du terrain. La ville informelle des "gueux" est un cloaque mais, tout en dénonçant l'iniquité des ségrégations urbaines, Lemebel met aussi en lumière la richesse humaine de ces espaces creux, interstitiels, révélant en négatif les lieux formels comme des espaces factices, superficiels, "superstitiels" ${ }^{29}$, villes de parade cachant la réalité des injustices sociales et le cynisme des politiques. Sans idéaliser le bidonville dont il est issu, Lemebel montre -dans une perspective typiquement postmoderne- que la ville informelle, même si elle représente un handicap social pour ses habitants, peut aussi être une école de la vie et de l'art. II en fait la démonstration dans son œuvre où toute l'écriture repose sur une esthétique du bricolage, de l'agglomérat de matériaux subculturels de récupération (citations de chansons populaires, etc.) et fluidité de la structure informelle.

Comme l'ont montré plusieurs sociologues, ethnologues et urbanistes, la géographie sociale des villes occidentales s'est modifiée au cours du XXe siècle: dans les années 1960 et 1970 notamment, les centres ont plus ou moins perdu leur statut hégémonique et I'on est passé de villes centralisées à des villes multifocales et polycentriques. La ville formelle "ombilicale" est donc devenue une sorte de coquille vide, espace de pure monumentalité ostentatoire. Par un effet de vases communiquants, certains centres se sont paupérisés, ont été partiellement "Squattés" ou envahis de façon ponctuelle par des populations marginales. Admirateur de Lemebel et militant queer comme lui, Sutherland s'est intéressé à la minorité homosexuelle. Libérée des contingences matérielles de la vie familiale (concentrée dans les cités dortoirs et les zones résidentielles) et recherchant l'anonymat protecteur des mégapoles, ces individus ont investi la ville formelle "molaire" en y développant-de façon parfois ponctuelle et transitoire- des pratiques informelles "moléculaires", l'informalité devenant plus que jamais -comme le soulignait Correa Díaz- un modus operandi. Sutherland montre que, contrairement à certains préjugés, ces usages ne sont pas seulement ludiques et consuméristes mais qu'ils peuvent aussi se comprendre comme une forme de résistance -consciente ou nonà la norme et fonctionner comme un véritable minage politique de I'honorabilité de la ville formelle par une pratique informelle. 


\section{NOTES}

1 Gran parte de lo que nos pasa es imaginario, porque no surge de una interacción real. [...] Los imaginarios han nutrido la historia de lo urbano. (C'est moi qui traduis).

2 Rosalba Campra est née à Córdoba, en Argentine, et elle enseigne la littérature hispano-américaine à l'université La Sapienza, à Rome. Elle est l'auteure de plusieurs essais (sur la littérature gauchesca, sur le tango...) et notamment sur les liens entre identité hispano-américaine et littérature.

3 C'est moi qui traduis toutes les citations extraites des œuvres de Sutherland. Selon une déclaration inédite d'Henri Billard, Sutherland pourrait plus ou moins se rapprocher d'un groupe d'écrivains "gays" dont le représentant le plus connu est le péruvien Jaime Bayly (1965).

4 Stéphanie Decante, comme beaucoup de spécialistes du Chili, pense qu'il vaut mieux parler de Transition vers la Démocratie plutôt que de Transition démocratique.

5 Traduit et cité par Correa Diaz, p. 23.

6 Le Lazarillo aurait été écrit entre 1540 et 1550. Titre français: La vie de Lazare de Tormès et de ses fortunes et adversités.

7 Titre complet: La vida del pícaro Guzmán de Alfarache, atalaya de la vida humana (Le gueux ou La vie de Guzman d'Alfarache guettechemin de vie humaine).

8 Titre complet: La vida del Buscón, llamado Pablos, ejemplo de vagamundos y espejo de tacaños (La vie de l'Aventurier don Pablos de Ségovie, vagabond exemplaire et miroir des filous).

9 Dans l'Espagne du Siècle d'Or (XVle-XVIle siècles), la "pureté de sang", indispensable pour occuper un rang élevé dans l'échelle sociale, était liée à l'absence d'ascendance juive ou musulmane.

10 Un pasillo estrecho con una ventana que da al muro de la fábrica. [...] Un comedor sin paredes donde las moscas, el sol y los variados tratos, expulsan sin tregua. Termina la casa en un pequeño patio, recinto donde se acumulan todas las escorias junto con las aguas sucias del palanganero y del lavabo. (C'est moi qui traduis.)

11 Pero el Zanjón, más que ser un mito de la sociología poblacional, fue un callejón aledaño al fatídico canal que lleva el mismo nombre. Una ribera de ciénaga donde a fines de los años cuarenta se fueron instalando unas tablas, unas fonolas, unos cartones, y de un día para otro las viviendas estaban listas. [...] Florecían entre las basuras las precarias casuchas. [...] siempre el asunto de la vivienda ha sido una excursión aventurera para los desposeídos [...] en el Zanjón, el agua para beber, cocinar o lavarse había que traerla de lejos, donde un pilón siempre abierto abastecía el consumo de la población callampa. Así también la evacuación de las aguas servidas y el alcantarillado se resumían en una acequia hedionda que corría paralela al rancherío, donde las mujeres tiraban los caldos fétidos del mojoneo. (C'est moi qui traduis.)

12 Mi nacimiento fue dentro del río Tormes, por la cual causa tomé el sobrenombre; [...] y estando mi madre una noche en la aceña, preñada de mí, tomóle el parto y parióme allí. De manera que con verdad me puedo decir nacido en el río. (C'est moi qui traduis.)

13 [...] inyectándome entonces el borde plateado de la orina que baja desnuda los peldaños hasta aposentarse en una estrella humeante. Yo me fumo esos vapores en un suspiro de amor por su exilio rebelde. (C'est moi qui traduis.)
14 Ahora hasta los lugares aborrecibles, al saberse ya perdidos, se convertían en sitios venerables. (C'est moi qui traduis.)

15 aprendí todo lo bueno y supe de todo lo malo: ces paroles sont probablement tirées du tango Las Cuarenta (1937), texte de Francisco Gorrindo et musique de Roberto Grela: Con el pucho de la vida apretado entre los labios, /la mirada turbia y fría, un poco lerdo el andar,/dobló la esquina del barrio y, curda ya de recuerdos,/ como volcando un veneno, esto se la oyó acusar:/Vieja calle de mi barrio donde he dado el primer paso,/vuelvo a vos gastado el mazo en inútil barajar,/con una llaga en el pecho, con mi sueño hecho pedazos,/que se rompió en un abrazo que me diera la verdad.// Aprendí todo lo malo,/aprendí todo lo bueno,/sé del beso que se compra,/sé del beso que se da [...]

16 Pareciera que en la evocación de aquel ayer, la tiritona mañana infantil hubiera tatuado con hielo seco la piel de mis recuerdos. [...] En ese revoltijo de olores podridos y humos de aserrín, "aprendí todo lo bueno y supe de todo lo malo", conocí la nobleza de la mano humilde y pinté mi primera crónica con los colores del barro que arremolinaba la leche turbia de aquel Zanjón. (C'est moi qui traduis.)

17 Dans un recueil de photos intitulé "Porquería visual" et intégré à Zanjón de la Aguada (pp. 158-161), Lemebel met une photo de "pitucos" du début du XXème siècle en regard avec celle d'une femme des bidonvilles de Santiago, vers la même époque.

18 Simón Bolívar (Caracas, 1783 - Colombie 1830): issu de l'aristocratie créole, il est le grand artisan de la guerre d'indépendance des colonies hispano-américaines contre les Espagnols, dans les années 1810-1820. II libère plusieurs colonies (dont les actuels Venezuela, Colombie, Pérou et Bolivie) et crée la Grande Colombie (Colombie, Equateur, Panama, Venezuela) mais doit renoncer au pouvoir en 1830. II avait reçu le titre de Libertador.

19 C'est moi qui souligne.

20 Que claro que le gustaría irse a vivir a otra parte y poner otro negocio. Pero que no se iba porque la Estación El Olivo era tan chica y todos los conocían y a nadie le llamaba la atención tan acostumbrados estaban. Ni los niños preguntaban porque nacían sabiendo. No hay necesidad de explicar, eso dijo la Japonesa y el pueblo se va a acabar uno de estos días y yo y usted con este pueblo de mierda que no pregunta ni se extraña de nada.

21 ¿Pero a qué demonios acarrear las víctimas (quiero decir muchachos) tan arriba en la montaña? ¿No era demasiado excéntrico del designio? Así es, en efecto, usted lo ha dicho: "excéntrico", fuera del centro. Los sacábamos a la periferia para cortarles todo vínculo con lo conocido, para aligerarlos del tabú, de la ciudad, de ropa.

22 Así nos resituamos en una ciudad diseminada, una ciudad de la que cada vez tenemos menos idea dónde termina, dónde empieza, en qué lugar estamos. (C'est moi qui traduis.)

23 L'expression "nouveaux libertins" est une allusion au roman de I'italien Pier Vittorio Tondelli, Altri libertini, Giangiacomo Feltrinelli Editore, 1980 (Les nouveaux libertins, traduction de Nicole Sels, Editions du Seuil, 1987).

24 Le mouvement gay a vu le jour aux Etats-Unis, en 1969, lors de la révolte de Stonewall où des milliers d'homosexuels new-yorkais s'étaient battus contre la police qui les harcelait sans cesse. Mais, après le militantisme de années 1970, la communauté gay est devenue de plus en plus discrète, en quête d'intégration et d'invisibilité. C'est alors qu'est apparu le mouvement queer, beaucoup plus politisé et ancré à 
gauche, critiquant le conformisme et le consumérisme de la communauté gay WASP et faisant cause commune avec les revendications des homosexuels pauvres et non blancs, des lesbiennes, des travestis, et des transgenres. Lemebel et Sutherland sont des militants queer.

25 Les lieux de déambulation de ces personnages sont: la cathédrale, le musée des Beaux Arts, le Mapocho, les places (plaza de Armas, plaza Italia, plaza Brasil), les parcs, les quartiers (Bellavista, Recoleta, Pío Nono), les gares (la Estación Central, Estación Los Héroes), le métro (la station Salvador del Metro), l'aéroport, les ponts (Puente Lira), les universités (I'Université du Chili, Diego Portales), les églises (Santo Domingo), mais aussi promenades, avenues et rues qu'ils parcourent sans cesse d'un bout à l'autre et en tous sens (paseo Ahumada, Avenida Providencia, avenida Santa María, avenida Portugal, calle Chacabuco, etc.).

26 [...] teníamos sexo ocasional por cualquier parte y a cualquier hora, por el Forestal, detrás de Bellas Artes a las cuatro de la mañana, en un conventillo del Barrio Brasil [...] o en el Metro de la Universidad de Chile, donde apasionadamente terminamos con un guardia azul en una de las salas de los andenes, ese tipo de espacios que nunca se utilizan para nada. (C'est moi qui traduis.)

27 El primer mes nos juntamos en el Prosit, en la Plaza Italia. [...] Terminábamos en el Parque Baquedano fumando pitos y en alerta de los cilones verdes. Los matorrales siempre nos ayudaron. Con el Axel tuvimos mucho sexo público [...] (C'est moi qui traduis.)

28 [...] la transmutación de una política abiertamente represiva en otra aparentemente consensual, marcada por lógicas neoliberales. (C'est moi qui traduis.)

29 Le "superstitiel" est un moyen publicitaire très en vogue sur Internet: il s'agit d'un cartouche publicitaire que l'internaute doit fermer avant de pouvoir accéder à la page qui l'intéresse. Chez les auteurs qui nous intéressent, nous pourrions dire que la ville formelle est "superstitielle" dans le sens où son hyper visibilité est un écran servant à cacher la réalité sociale.

\section{BIBLIOGRAPHIE}

ARENAS, R. (2001). El palacio de las blanquísimas mofetas. Barcelona: Tusquets editores.

CAMPRA, R. (1994). "La ciudad en el discurso literario". Sic, N 5.

CHAUCHADIS, C. (2006). "L'apprentissage de l'honneur dans le Lazarillo de Tormes". Sanchez, J. P. (coordinateur). Le Roman picaresque (pp. 133-146). Editions du Temps.

CORREA DIAZ, A. C. (2007). Réflexion sur les changements introduits par la formalisation des quartiers informels en Amérique latine. Mémoire de Master 2 "Urbanisme et territoires» mention "urbanisme», dirigé par Jean-Claude Driant, Institut d'Urbanisme de Paris, université Paris XII - Val de Marne, disponible en ligne en version pdf.

-_-. (2012). La formalisation des quartiers informels en Amérique latine, Editions Universitaires Européennes.

DECANTE, S. (2005). «La loca geografía de Pedro Lemebel, De las memorias de la ciudad a la memoria de los escritos de la ciudad". Orecchia-Havas (Ed.), Mémoire(s) de la Ville dans les mondes hispaniques et luso-brésiliens (pp. 67-77). Bern: Peter Lang.

DELEUZE, G., GUATTARI, F. (1980). Mille plateaux, Capitalisme et schizophrénie 2. Paris: Les éditions de Minuit.
DONOSO, J. (1987). El lugar sin límites. Barcelona: Biblioteca Breve, Seix Barral.

_-_. (1999). Ce lieu sans limites (traduction d'Aline Schulman). Paris: Le Serpent à plumes.

ESTEVEZ, A. (1997). Los Palacios distantes. Barcelona: Tusquets Editores.

GARCÍA CANCLINI, N. (2005). Imaginarios urbanos. Buenos Aires: Eudeba, Editorial Universitaria de Buenos Aires.

GRACQ, J. (1985). La Forme d'une ville. Paris: José Corti.

HARDOY, J., SATTERTHWAITE, D. (1981). “Shelter” Need and Response. London, NY, Toronto, Chichester, Brisbane: Ed. John Wilwey and Sons.

LEMEBEL, P. (2004). La esquina es mi corazón. Santiago de Chile: Seix Barral / Biblioteca Breve.

_-_. (2004). Zanjón de la Aguada, Santiago de Chile: Seix Barral / Biblioteca Breve.

LÓPEZ GARCÍA, I. (2007). La question du genre dans les chroniques de Pedro Lemebel. Thèse de Doctorat Nouveau Régime dirigée par Milagros Ezquerro, Université Paris Sorbonne. Récupéré de http://www.paris-sorbonne.fr/fr/ IMG/pdf/LOPEZ-GARCIA_position.pdf.

MOULIAN, T. (1998). Chile actual: anatomía de un mito, Santiago: LOM.

PUIG, M. (1972). Boquitas pintadas. Barcelona: Seix Barral.

-_-. (1972). Le plus beau tango du monde, traduction française de Laure Guille-Bataillon Paris: Éditions Denoël.

RICHARD, N. (1998). Residuos y metáforas. Santiago: Cuarto Propio.

RICO, F. (Ed.). (1990). Lazarillo de Tormes. Madrid: Ediciones Cátedra.

ROLIN, O. (1988). Sept villes. Paris: Rivages.

SOUILLER, Didier. "Corrigé de l'épreuve de littérature générale et comparée", Rapport sur la session 2005 de l'Agrégation de Lettres Modernes. Strubel, A. (Ed.). Récupéré de ftp://trf.education.gouv.fr/pub/edutel/siac/ siac2/jury/2005/agreg_ext/let_mod.pdf

-_-. (1980). Le roman picaresque. Paris: PUF.

SOUQUET, L. (2005). «Viaje a La Havana de Reinaldo Arenas: «l'essence du temps localisée" et le "corps sans organes" comme mémoire de la ville." Orecchia-Havas (Ed.), Mémoire(s) de la Ville dans les mondes hispaniques et luso-brésiliens (pp. 111-119). Bern: Peter Lang.

---. (2007). "Ángeles negros de Juan Pablo Sutherland, des marges au centre: les lieux sans limites des "anges déchus" de la ville postmoderne". Orecchia Havas, Teresa (Ed.), Les Villes et la fin du XXe siècle en Amérique latine: Littératures, cultures, représentations (pp. 299-324), Bern: Peter Lang.

---. (2009). "Ligne de fuite néo-picaresque dans l'autofiction hispanoaméricaine: Arenas, Copi, Lemebel, Vallejo". Hommage à Milagros Ezquerro, Théorie et fiction (pp. 601-622). México / París: RILMA 2 / ADEHL.

SUTHERLAND, J. P. (compilador). (2001). A corazón abierto, Geografía literaria de la homosexualidad en Chile. Santiago de Chile: Señales/antología, Editorial Sudamericana.

SUTHERLAND, J. P. (2004). Ángeles negros. Santiago de Chile: ediciones Metales Pesados.

VALLEJO, F. (2004). El fuego secreto, Bogotá: Alfaguara.

VIRILIO, P. (1976). L'insécurité du territoire. Paris: Stock.

$>$ 